\section{Embryological cookery book}

Methods in Mammalian Reproduction. Edited by J. C. Daniel. Pp. 566. (Academic: New York, San Francisco and London, 1978.) \$39.50; £25.65.

TExTs that tell you how to do things, whether they be cookery books or manuals of bee-keeping or motorcycle maintenance, can be valuable both for the experienced and for the novice; they also have a particular fascination for the voyeur, who has no intention of actually keeping bees or maintaining motor-cycles, but likes to identify with the contortions of those who do. Even the best of such books can be dangerous, however, if they are accepted as an alternative to visiting the master, watching him perform, and then trying the procedure yourself under his critical eye.

With this caveat, Methods in Mammalian Reproduction is warmly to be welcomed. Its predecessor, Methods in Mammalian Embryology, which appeared in 1971 with the same editor but a different publisher, must have achieved an extremely high average Citation Index rating; my own copy is constantly borrowed. The present volume has a very similar mix of 'developmental' and 'reproductive' topics to the previous one, despite its title, but the themes are now a little more sophisticated. For example, induction of ovulation has been succeeded by in vitro oocyte maturation, fertilisation by parthenogenesis, aggregation chimeras by injection chimeras.

Most of the book is concerned with embryos and the remarkable things that can be done to them during the preimplantation period (oocyte maturation, experimental parthenogenesis, manipulation of ploidy, injection chimeras, immunological interference with development) or subsequently (embryo culture during and after implantation. embryo grafts to the chick, limb: regeneration). Others describe techniques that can be applied more generally during development (freezing, tissue separations, one- and two-dimensional gel clectrophoresis, and fluorography). Then there are chapters on particular groups of animals (marsupials; primates; embryo culture in rabbits, sheep and cows; embryo transfer in farm animals) Finally, there are a few rather heterogeneous chapters on the maternal side, dealing with pheromones, experimental delay of implantation, female genital tract secretions, intrauterine devices in monkeys and rodents, grafting endometrium to ectopic sites, laparoscopy (not in humans) and amniocentesis (only in humans).

Some chapters are long and very detailed: gel electrophoresis; injection chimeras; amniocentesis, including cell culture and chromosome banding. Some read more as interesting accounts of rather esoteric research fields than as descriptions of widely used techniques: endometrial transplantation; immunological inhibition of development (mainly with antisera against zona pellucida); regeneration of mammalian limbs (they don't, even with electrical stimulation, but bits of brain implanted in the stump help, and bone, muscle and nerve individually do quite well).

Bibliographies are satisfactory only up to 1976, with some 1977 papers listed as being in press; the descriptions of some rapidly advancing areas, such as the use of ultrasound and amniocen-

\section{Dioxin workshop}

Dioxin: Toxicological and Chemical Aspects. Edited by F. Cattabeni, A. Cavallaro and G. Galli. Pp.222. (SP Medical and Scientific Books/ Spectrum: New York and London; 1978.) Distributed by Halsted/Wiley. $£ 14.50 ; \$ 26.50$.

ON 10 July, 1976 the north Italian town of Séveso was contaminated with the discharge from a chemical reactor used for the manufacture of trichlorophenol. The discharge consisted primarily of sodium trichlorophenate, but also contained an extremely toxic byproduct of the reaction 2,3,7,8-tetrachlorodibenzodioxin (TCDD).

Faced with a serious pollution problem the Province of Milan and the Lombardy Regional Government sponsored a workshop in October 1976 to which scientists working on polychlorinated dibenzodioxins (PCDDs) were invited. This book records the workshop proceedings.

Research on the toxicology of the PCDDs has increased at an exponential rate since 1976. Recently published results, for example, have shown. that TCDD will cause tumours in experimental animals. As the workshop proceedings contain no discussion of the potential carcinogenicity of the dibenzodioxins and make only cursory reference to mutagenicity studies, its coverage of the toxicology of these compounds is incomplete.

That section of the book dealing with the chemistry is, nevertheless, useful. Accounts are given of methods currently in use for the analysis of environmental samples containing PCDDs by various combinations of gas liquid chromatography, mass spectrometry and mass fragmentography; and there is some discussion of the prob- tesis in prenatal diagnosis, tend in consequence to be a little dated. Editing could be improved: for example, techniques for the recovery of embryos from monkey reproductive tracts are distributed among three chapters, laparascopy, IUDs, and primates; no cross-references are given. The index is inadequate.

These are minor criticisms. If you have found Joe Daniel's $M M E$ useful, you will want to possess $M M R$ also. The initial purchase price is high, but you are likely to recoup it many times over, by increased efficiency, avoidance of costly errors, and some appreciation of what you may be letting yourself in for.

Anne McLaren

Anne McLaren is Director of the MRC Mammalian Development Unit, London, $U K$

lems encountered in separating dibenzodioxins from other industrial pollutants (for example, polychlorinated biphenyls).

But perhaps of most interest will be the information on decontamination methods following TCDD pollution. Both incineration and photolysis are considered. Temperatures in excess of $800^{\circ} \mathrm{C}$ are necessary for the destruction of TCDD-at lower temperatures $\left(300-500^{\circ} \mathrm{C}\right)$ more of the dibenzodioxin is formed from precursors of TCDD and 2,4,5-trichlorophenate.

The processes of photolysis are covered in four separate chapters. In the presence of a hydrogen donor such as methanol, ultraviolet irradiation of TCDD will selectively remove chlorine atoms from the molecule, thus rendering it less toxic. Alternative solvents/hydrogen donors are required for field conditions, and the action of two such combinations (ethyl oleate and xylene, olive oil and cyclohexanone) is described. Results reported from field trials at Séveso suggest that the olive oil/cyclohexanone method effectively promotes the degradation of TCDD, and that little of the dibenzodioxin was leached into the soil, despite heavy rain during the experiment.

Two potential decontamination methods not referred to are chlorinolysis and bacterial action. Their omission is not particularly serious; however, as bacteria are probably responsible for the detoxification of most of the TCDD in the environment these are organisms which cannot be ignored.

On the whole the book should prove useful as an introductory text for students of the dibenzodioxins.

Alastair Hay

Alastair Hay is Research Fellow in the Department of Animal Physiology and Nutrition at the University of Leeds, UK. 